\title{
stom

\section{NDE of metal damage: ultrasonics with a damage mechanics model}

\author{
Hung-Yang Yeh *, Jung-Ho Cheng * \\ Department of Mechanical Engineering, National Taiwan University, No. 1, Sec. 4, Roosevelt Road, Taipei 106, Taiwan, ROC
}

Received 16 August 2003; received in revised form 16 August 2003

\begin{abstract}
This research describes a nondestructive method for the quantitative estimation of property variations due to damage in metal materials. The method employs a damage mechanics model, which accounts for stiffness degradation and damage evolution of a metal medium with a measurement of ultrasonic velocity. In order to describe the progressive deterioration of materials prior to the initiation of macrocracks, we have developed a new damage mechanics model. Thereafter, a finite element model valid for numerically describing such damage process has been developed by ABAQUS/Standard code, and correlations between damage state, elastic stiffness and plastic strain could be found by the results of the finite element simulation. The property variations due to damage evolution are calculated based on the Mori-Tanaka theory, and then the ultrasonic velocity can be predicted by Christoffel's equation. When the measured velocity is coupled with the theoretically predicted velocity, the unknown damage variable is solved, from which other residual properties are determined by the predictions of damage model. The proposed technique is performed on type 304 stainless steel bars. The numerical results obtained by the simulation were compared with experimental ones in order to verify the validity of the proposed finite element model and good agreement was found. It is shown that the damaged properties of metals can be estimated accurately by the proposed method.
\end{abstract}

(C) 2003 Elsevier Ltd. All rights reserved.

Keywords: Damage mechanics model; Nondestructive testing (NDT); Finite element model

\section{Introduction}

Microvoids and microcracks may arise in the course of metal production, and further loading will enlarge and coalesce these microdefects. Experimental results show that such a kind of damage can cause the degradation of stiffness, strength, and working life of engineering materials. In order to describe the gradual or progressive deterioration of mechanical properties and to predict the final failure, many researchers have devoted themselves to the development of a damage mechanics model.

Kachanov (1958) has been considered the pioneer of continuum damage mechanics (CDM) since he published the first paper on a field variable $\psi$ called continuity. About 15 years later the concept of the

\footnotetext{
${ }^{*}$ Corresponding authors. Tel./fax: +886-2-2362-6935 (H.-Y. Yeh), Tel.: +886-2-2362-2191; fax: +886-2-2363-1755 (J.-H. Cheng).

E-mail addresses: f87522504@ntu.edu.tw, f87522504@ms87.ntu.edu.tw (H.-Y. Yeh), jhcheng@ntu.edu.tw (J.-H. Cheng).
} 
damage variable $D=1-\psi$ was proposed on the basis of thermodynamics: $0 \leqslant D \leqslant 1$ ( 0 for the original state and 1 for failure). During these 15 years, the concept of effective stress was introduced by Rabotnov (1968) for the uniaxial load and extended to the general case by Lemaitre (1971) and Chaboche (1979). Based on the damage variable and the concept of effective stress, constitutive equations of evolution were developed to predict the initiation of macrocracks for practical applications. In the 1980s, damage theory was developed more rigorously based upon thermodynamics and micromechanics, and was begun to apply to engineering. Leckie and Hayhurst (1974) and Hult (1974) used the theory to solve creep problems. While Truesdell and Noll (1965), Jaunzemis (1967), Malvern (1969), and Maugin (1992) worked out a systematic formulation of inelastic constitutive equations by the irreversible thermodynamics theory, Krajcinovic and Lemaitre (1987), Lemaitre and Chaboche (1990), and Lemaitre (1985, 1992), successfully applied this approach to problems of damage.

Nondestructive testing (NDT) methods play an important role in the physical characterization of engineering materials and in the assessment of their quality and serviceability in structures. In a wide range of different NDT methods, ultrasonic velocity approaches have been used extensively to evaluate the density and the elastic constants of metals and alloys. When an ultrasonic wave propagates in a material, the magnitude of the velocity is related to the density and the stiffness of the material. Since material properties depend on damage, correlations can be established between ultrasonic velocity and damage, such that ultrasonic velocity methods can be utilized to monitor the damage evolution of materials. Nagarajan (1971) and Panakkal et al. (1984) found linear correlations between these quantities for polycrystalline alumina and sintered fuel pellets, respectively. Gruber et al. (1988) combined ultrasonic velocity measurements and micromechanics model to estimate the local property variation in porous ceramics. Jeong and Hsu (1996) proposed a method by means of composite micromechanics and ultrasonic velocity to quantitatively estimate material properties of porous ceramics. Augereau et al. (1999) demonstrated the ability of acoustic signature technique to detect in a nondestructive way mechanical property variations due to the damage of the internal material structure for 304L steel samples. They asserted that this technique provided velocity measurement with an accuracy to $1 \%$.

The aim of the present work is to propose a nondestructive, quantitative, and mechanics-based damage evaluation technique. This technique combines the nondestructive ultrasonic pulse-echo method with a damage mechanics model. By measuring the ultrasonic L-wave velocity of the damaged sample and comparing with the theoretically calculated velocity value, we can obtain the current damage value and residual mechanical properties from the finite element simulation. To achieve our purpose, we construct a new damage model, defining a physically meaningful damage variable and accounting for the damage effects on mechanical properties using the homogenization method. Then we develop a finite element model valid for numerically describing this damage model. By using Christoffel's equation, we correlate the ultrasonic testing method with the damage mechanics model. The proposed damage characterization technique has been successfully applied to type 304 stainless steel specimens strain-damaged by uniaxial tension.

\section{Analysis procedure}

Fig. 1 illustrates the schematic diagram of the approach used. The key factor of this technique is the gradual degradation in the elastic stiffness as a result of damage growth, since it connects the prediction of the damage mechanics model with nondestructive testing results. Fig. 2 shows the concept of damaged elasticity. To give an example, Young's modulus of the original stainless steel 304 samples is about 200 $\mathrm{GPa}$. When the undamaged samples are loaded to the plastic regime, the induced damage will lower Young's modulus to $180 \mathrm{GPa}$ or even smaller. Because the elastic ultrasonic wave propagates in the medium depending on the elastic constants, the lower the elastic constants of the medium, the slower the 


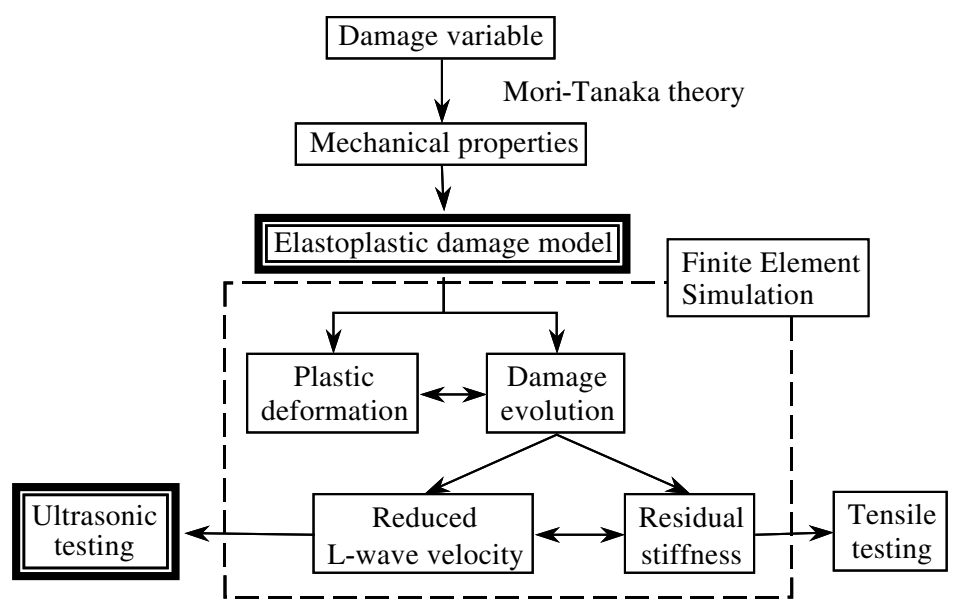

Fig. 1. Procedure for assessing metal damage.

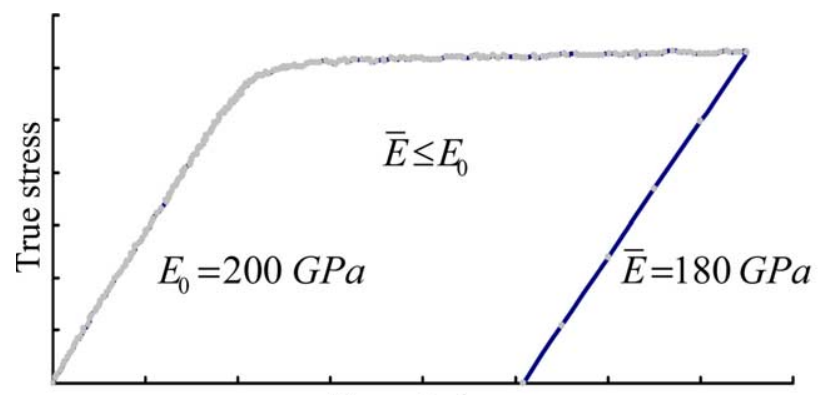

True strain

Fig. 2. Damaged elasticity (take SUS 304 samples as an example).

ultrasonic wave velocity becomes. In order to describe the progressive deterioration of materials prior to the initiation of macrocracks, we developed a damage model. First, we defined a physically meaningful damage parameter as volume fraction of microvoids in a unit cell. As a result, the influence of damage on elasticity could be estimated using a micromechanical homogenization method. Secondly, we established an elastoplastic damage model, which adopted the yield function of mildly voided metals as proposed by Gurson (1977), to describe the deformation and damage evolution processes of ductile materials. Subsequently, a finite element model which adopted this damage model was constructed by ABAQUS/Standard code, and correlations between the damage state, elastic stiffness, and plastic strain could be found from the results of finite element simulation. In order to verify the proposed damage model, we performed tensile testing on type 304 stainless steel specimens to measure the residual Young's moduli of the strain-damage samples and compared the numerical results with the experimental values. Furthermore, we adopted Christoffel's equation to correlate the elastic constants with the ultrasonic velocity of longitudinal wave (L-wave). Then, we chose ultrasonic pulse-echo method to evaluate L-wave velocities of stainless samples under certain loading. Since the correlations between damage value, plastic deformation, residual stiffness and reduced Lwave velocity were established from the finite element results, by coupling the ultrasonic testing results with the predictions of the elastoplastic damage model, we could nondestructively and quantitatively evaluate the metal damage. 


\section{Elastoplastic damage model}

This study aims at establishing an elastoplastic damage model. The metal damage appears in the form of microvoids. Before the derivation of theoretical equations, the characteristics of the voids are assumed to be of the same size, sphere-shaped, isotropic, and randomly distributed. Accordingly, the overall mechanical properties will be isotropic. A complete elastoplastic damage model involves seven components: (1) damage variable; (2) effective elastic constants; (3) constitutive law of elastic deformation; (4) yield criterion; (5) constitutive law of plastic deformation; (6) damage evolution law; and (7) failure criterion. Details of each component are described in the following sections.

\subsection{Damage variable}

Fig. 3 shows a unit cell with spherical voids. Let $V$ be the volume of the unit cell and $V_{\text {c }}$ the overall volume of voids. The damage variable $D$ is defined as the volume fraction of voids as follows:

$$
D=\frac{V_{\mathrm{c}}}{V}
$$

In case the volume of voids equals zero, i.e., $D=0$, we have a perfect material.

\subsection{Constitutive law of elastic deformation}

Generalized Hooke's law was adopted to describe the linearly elastic deformation of metals. To take the damage state into account, we modified the original stiffness tensor $C_{i j k l}$ into the damaged stiffness tensor $\bar{C}_{i j k l}(D)$. For an isotropic material, the stress-strain relation of elastic deformation is given by

$$
\sigma_{i j}=\bar{C}_{i j k l}(D) \varepsilon_{k l}^{\mathrm{e}}=2 \bar{G} \varepsilon_{i j}^{\mathrm{e}}+\left(\bar{K}-\frac{2}{3} \bar{G}\right) \varepsilon_{k k}^{\mathrm{e}} \delta_{i j}
$$

where $\bar{G}, \bar{K}$ are effective shear modulus and effective bulk modulus, respectively. They both depend on the damage variable and can be estimated using the homogenization methods. Details will be described in the next section.

\subsection{Effective elastic constants: Mori-Tanaka method}

By using the Mori-Tanaka method (1973), $\bar{G}$ and $\bar{K}$ can be expressed as functions of damage variable,

$$
\bar{G}=G_{0}\left[1-\frac{15\left(1-v_{0}\right)}{\left(7-5 v_{0}\right)+2 D\left(4-5 v_{0}\right)} D\right], \quad \bar{K}=K_{0}\left[1-\frac{3\left(1-v_{0}\right)}{2\left(1-2 v_{0}\right)+D\left(1+v_{0}\right)} D\right],
$$

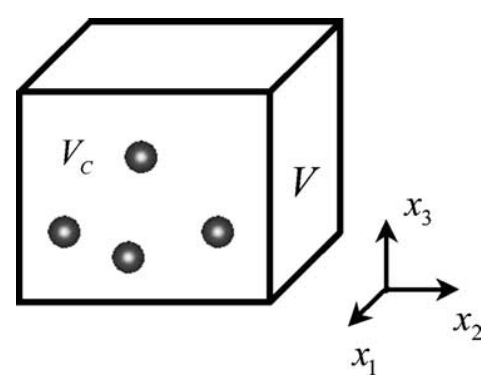

Fig. 3. Unit cell with spherical voids. 


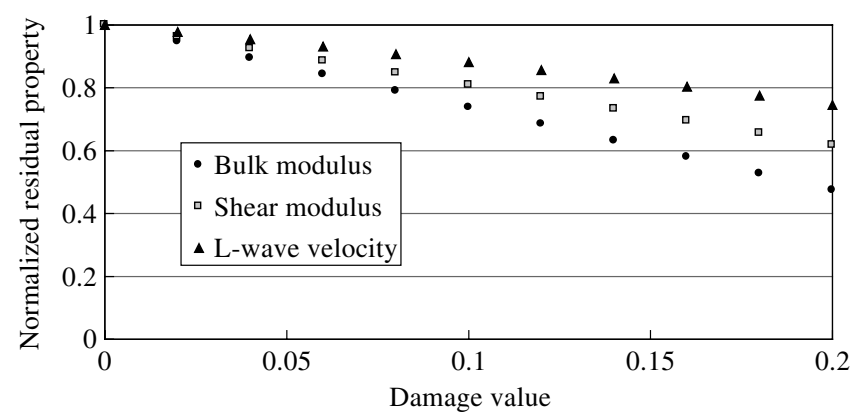

Fig. 4. Normalized residual stiffness and L-wave velocity.

where $G_{0}$ and $K_{0}$ are the shear modulus and bulk modulus of perfect materials, respectively. Fig. 4 illustrates how elastic constants decrease with the damage variable.

\subsection{Constitutive law of plastic deformation}

For a metal containing a dilute concentration of voids, Gurson (1977) proposed a formula based on a rigid-plastic upper bound solution for spherically symmetric deformations of a spherical void in a deformed solid. This criterion combines the effects of both the deviatoric part and the hydrostatic component, and is represented as follows:

$$
\phi=\left(\frac{\bar{\sigma}}{\sigma_{\mathrm{y}}}\right)^{2}+2 q_{1} f \cosh \left(\frac{3 q_{2} \sigma_{\mathrm{H}}}{2 \sigma_{\mathrm{y}}}\right)-\left(1+q_{3} f^{2}\right),
$$

where $\bar{\sigma}=\sqrt{\frac{3}{2} s_{i j} s_{i j}}$ is von Mises equivalent stress, $\sigma_{\mathrm{y}}$ is the yield stress of the matrix, $\sigma_{\mathrm{H}}$ is hydrostatic stress, $f$ is the volume fraction of the voids in the material. Tvergaard (1981) introduced three constants $q_{1}, q_{2}$, and $q_{3}$ to make the predictions of the Gurson model agree with numerical studies of "ordered" voided materials in plane strain tensile fields; one can recover the original Gurson model by setting $q_{1}=q_{2}=q_{3}=1$.

Using the associated flow law, the plastic strain rate is expressed as

$$
\dot{\varepsilon}_{i j}^{\mathrm{p}}=\dot{\lambda}_{\mathrm{p}} \frac{\partial \phi}{\partial \sigma_{i j}}=\dot{\lambda}_{\mathrm{p}}\left(\frac{1}{3} \frac{\partial \phi}{\partial \sigma_{\mathrm{H}}} \delta_{i j}+\frac{3}{2 \bar{\sigma}} \frac{\partial \phi}{\partial \bar{\sigma}} s_{i j}\right),
$$

where $\dot{\lambda}_{\mathrm{p}}$ is the plasticity associated multiplier and $s_{i j}$ is the deviatoric stress tensor.

\subsection{Damage evolution law}

The damage increment consists of the growth of existing voids and the nucleation of new cavities. The growth of existing voids is formulated based on the conservation law of mass and is expressed as

$$
\dot{D}_{\text {growth }}=(1-D) \dot{\varepsilon}_{k k}^{\mathrm{p}},
$$

where the dummy index implies the summation convention. From the above equation we can deduce that the damage increases under positive mean plastic strain state.

The nucleation of voids can occur due to the microcracking and decohesion of the particle-matrix interface. According to the model proposed by Chu and Needleman (1980), the evolution of nucleated voids is given with the relation to the effective plastic flow of the matrix: 


$$
\dot{D}_{\text {nucleation }}=A \dot{\bar{\varepsilon}}_{\mathrm{m}}^{\mathrm{p}}
$$

where

$$
A=\frac{f_{\mathrm{n}}}{s_{\mathrm{n}} \sqrt{2 \pi}} \exp \left[-\frac{1}{2}\left(\frac{\bar{\varepsilon}_{\mathrm{m}}^{\mathrm{p}}-\varepsilon_{\mathrm{n}}}{s_{\mathrm{n}}}\right)^{2}\right] .
$$

The nucleation function $A / f_{\mathrm{n}}$ is assumed to have a normal distribution, where $A$ is the coefficient and $f_{\mathrm{n}}$ is the volume fraction of void nucleating particles. The normal distribution of the nucleation strain has a mean value $\varepsilon_{\mathrm{n}}$ and a standard deviation $s_{\mathrm{n}}$. Voids are nucleated only in tension. The mechanism of void nucleation, therefore, will not be considered as long as materials are in the compressive stress state.

\subsection{Failure criterion}

The term failure is defined as the initiation of the first macrocrack. According to Lemaitre's suggestion, the material failure can be specified when the damage variable $D$ reaches a critical value $D_{\mathrm{c}}$. The value of $D_{\mathrm{c}}$ of any kind of material must be acquired by a series of careful experiments. Brown and Embury (1973) suggested that the critical damage be 0.15 , while Tvergaard and Needleman (1984) suggested that the critical damage range from 0.15 to 0.25 for ductile materials.

\section{Correlation between ultrasonic velocity and stiffness}

According to Christoffel's equation, the correlation between the ultrasonic velocity of L-wave and the elastic constants is expressed as

$$
v_{\mathrm{L}}=\sqrt{\frac{\lambda+2 \mu}{\rho}}=\sqrt{\frac{3 K+4 G}{3 \rho}},
$$

where $\lambda, \mu$ are Lamé's constants, $K$ is bulk modulus, $G$ is shear modulus, and $\rho$ is the density of the material. The relation between damage value and the degraded L-wave velocity is illustrated in Fig. 4 (triangular symbols). In the viewpoint of theoretical modeling, the reduction in the L-wave velocity is smaller than that in the elastic constants. Although the variation in the L-wave velocity is less significant, the decreasing trend is quite obvious. On the other hand, as pointed out in the literatures (Birks et al., 1991; Augereau et al., 1999), the ultrasonic velocity method is well known for its high accuracy. Even a slight change in ultrasonic velocity can represent mechanical property variations of materials with accuracy.

\section{Materials and experiments}

This section describes the material and experimental aspects. In order to verify the applicability of the proposed technique, two methods are used to detect the damage state, with one being destructive and the other nondestructive. The former refers to the measurement of the elastic modulus, while the latter refers to that of the ultrasonic wave speed.

\subsection{Specimen preparation}

The material consisted of commercially available stainless steel 304 bars. The originally cylindrical bars were machined to the profile suggested by ASTM E 8. Fig. 5(a) shows the shape and size of the tensile 


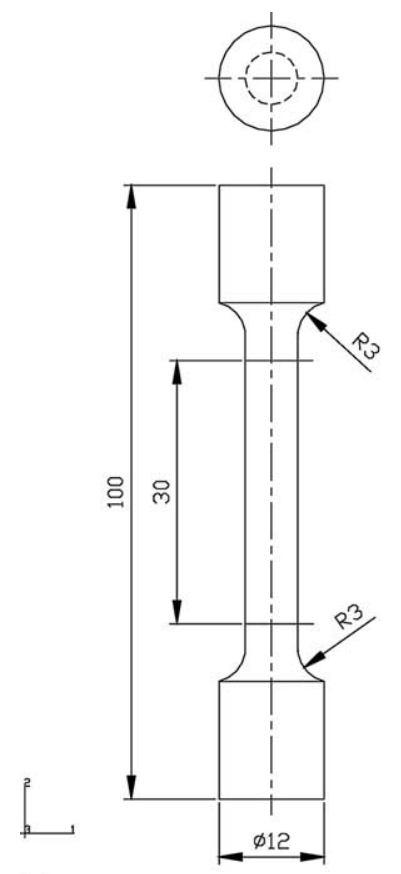

(a)

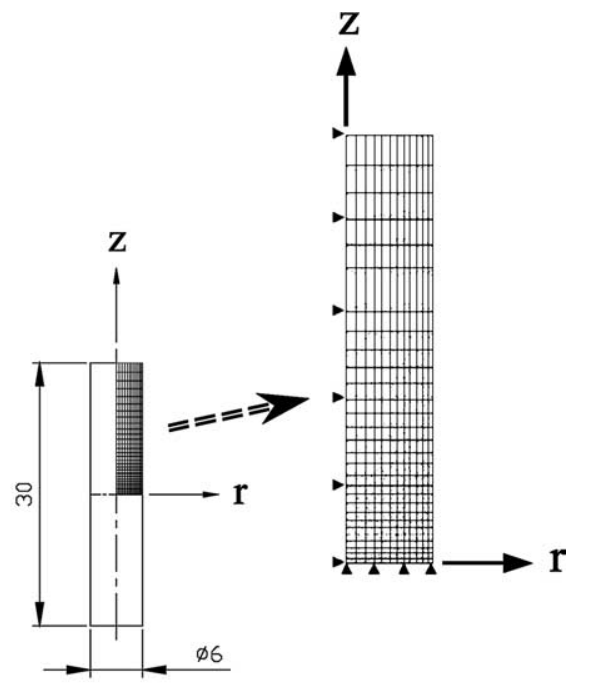

(b)

Fig. 5. (a) Specimen geometry, (b) finite element mesh.

specimen. In order to eliminate residual stress resulting from cold working, all bars were annealed for $1 \mathrm{~h}$ at $1100{ }^{\circ} \mathrm{C}$.

\subsection{Uniaxial tension tests}

Uniaxial tensile tests were performed on a universal testing machine at the speed of $0.05 \mathrm{~mm} / \mathrm{s}$. The true axial stress-strain curves are presented in Fig. 6 . The curve can be best fitted by using a power-law equation

$$
\sigma=k \varepsilon^{\mathrm{n}} .
$$

The main mechanical properties of 304 stainless steel bars are listed in Table 1 . These are the necessary data for use in the finite element simulation.

After the fundamental mechanical properties were obtained from the uniaxial tension test, the residual elastic constants of the strain-damaged specimens were measured. Owing to the influence of damage on elasticity, Young's moduli of the damaged specimens are lower than that of the original material. In order to investigate the trend of the degradation in elastic constants, a series of tension tests with different strokes were performed. Every loading stroke was exerted on an individual tensile specimen. Next, all specimens were unloaded and the damaged Young's moduli were measured one by one by using strain gauges. The results of the degraded Young's moduli (normalized by the initial Young's modulus) against different values of the equivalent strain are shown in Fig. 9 (triangular symbols) and will be further discussed in Section 6.2.1.

\subsection{Measurement of ultrasonic velocity}

In order to nondestructively evaluate the damage state and compare with the results obtained from the damage mechanics model, the ultrasound velocities in the loaded 304 stainless steel specimens were 


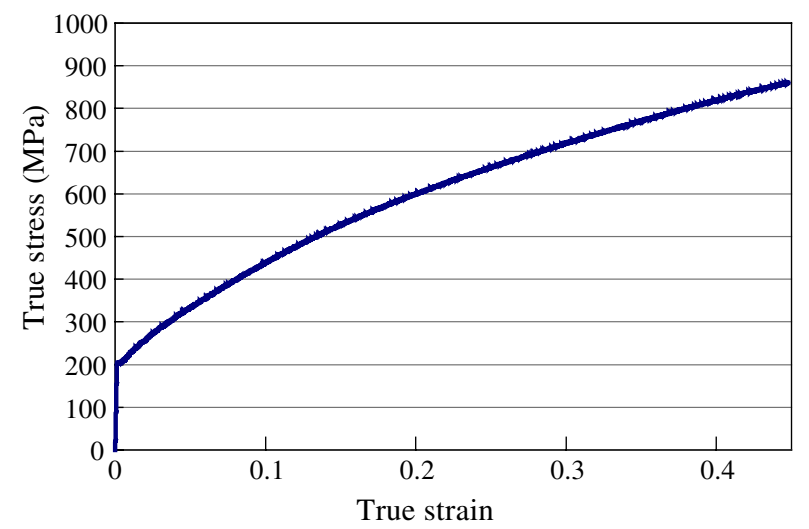

Fig. 6. True axial stress-strain curve of 304 stainless steel.

Table 1

Mechanical properties of 304 stainless steel

\begin{tabular}{lllll}
\hline$E^{\mathrm{a}}(\mathrm{GPa})$ & $v^{\mathrm{b}}$ & $\sigma_{\mathrm{y}}{ }^{\mathrm{c}}(\mathrm{MPa})$ & $K^{\mathrm{d}}(\mathrm{MPa})$ & $n^{\mathrm{d}}$ \\
\hline 200 & 0.29 & 205 & 1207 & 0.43 \\
\hline
\end{tabular}

${ }^{\text {a }}$ Young's modulus.

${ }^{\mathrm{b}}$ Poisson ratio.

${ }^{\mathrm{c}}$ Yield stress.

${ }^{\mathrm{d}}$ Parameters of the power law (10).

measured in the axial direction using a pulse-echo method. We prepared the ultrasonic testing samples by cutting off one part of thickness $d$ from each loaded specimen and polishing the surface. Thereafter, we measured the ultrasound velocities of the cut and polished samples.

Because our goal is to develop an engineering applicable approach, we employ the portable $A$-scan equipment to inspect the prepared samples. The measurements were made with a broadband ultrasonic pulse, and the data were obtained in the time domain (Fig. 7). A straight beam contact transducer of 10 $\mathrm{MHz}$ center frequencies was used. The radiofrequency waveforms of various pulses were captured and

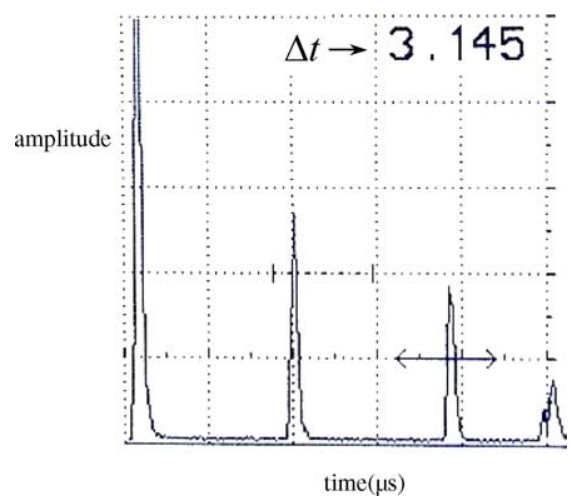

Fig. 7. Time domain chart of ultrasonic pulses. 
stored in an oscilloscope, and the time-of flight difference for calculating the velocity was measured by a peak detection method. Time-of-flight measurements were made with the aid of two time gates that defined the boundaries of the time domain area of interest. To reduce the effect of ultrasonic noise, only signals above a preset threshold were measured. The time-of-flight difference $\Delta t$ was obtained by calculating the time difference between two consecutive back surface echoes. The 304 stainless steel sample was assumed to be nondispersive over the frequency range used. The ultrasonic velocity was then given by $V=2 d / \Delta t$, where $d$ is the sample thickness. The results of the ultrasonic L-wave velocities against different values of the equivalent strain are shown in Fig. 10 (square symbols) and will be further discussed in Section 6.2.2.

\section{Finite element simulations}

\subsection{Finite element model}

The commercial finite element program ABAQUS/Standard was adopted to model and simulate the tensile deformation process of stainless steel 304 bars. ABAQUS was developed by Hibbitt, Karlsson and Sorensen (HKS), Inc. This program was developed on the basis of implicit integration method; therefore, Newton's iteration process was needed.

Because the tensile bars are round in shape, a quarter model with axisymmetric elements was constructed. The finite element mesh is shown in Fig. 5(b), in which a symmetric boundary condition is defined on the $r$-axis and $z$-axis is the axisymmetric axis.

The fundamental mechanical properties, including the initial elastic constants and the plastic parameters, for use in the simulation are given in Table 1. Because the elastic constants change depending on the damage variable, we introduced the concept of field variable to deal with their variation. In ABAQUS, we defined the field variable as the damage variable and employed the "USDFLD" option to implement the degradation of the elastic constants discussed in Section 3. The user subroutine "USDFLD" was originally empty. We wrote the stiffness degradation law (Eq. (3)) in the subroutine. The procedure of the simulation that joins the user subroutine is illustrated in Fig. 8. At the beginning of every time increment, ABAQUS acquired the field variable via the user subroutine USDFLD. The values of the field variable defined in this

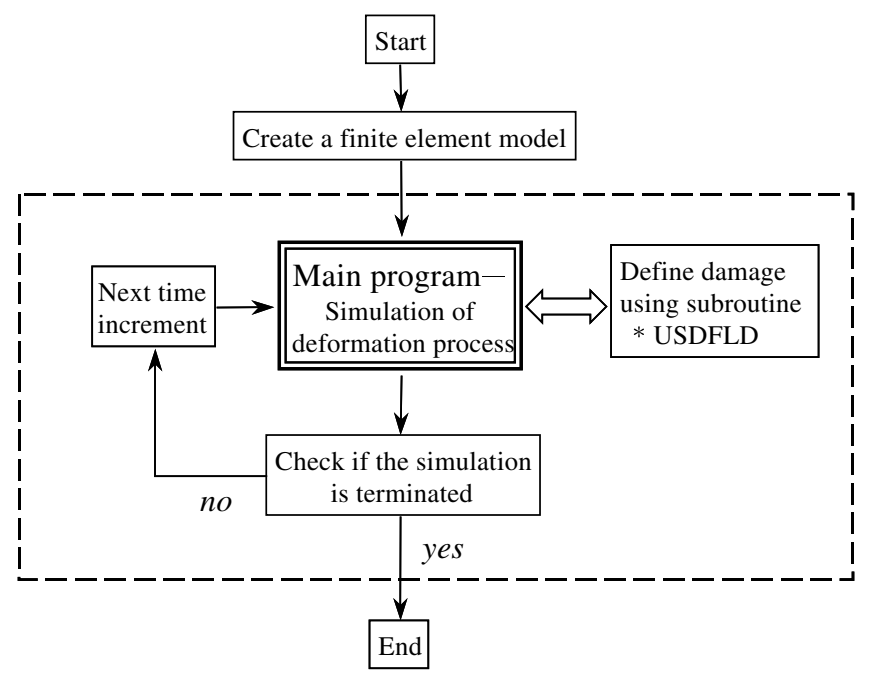

Fig. 8. The flow chart of the computational process of ABAQUS. 
routine were used to calculate the values of material properties that were defined based on the field variable. After the material properties at each integration point were ascertained, ABAQUS started the calculation of that time increment.

The plastic deformation and the damage evolutional process defined in Section 3 were computed in the main program of the finite element model. ABAQUS offered an inelastic material model, which was based on Gurson's porous metal plasticity theory (Gurson, 1977) with void nucleation, to model materials with a dilute concentration of voids in which the relative density was greater than 0.9. This model defined the inelastic flow of the porous metal on the basis of a potential function that characterized the porosity in terms of a single state variable, the relative density. The Tvergaard parameters $q_{1}, q_{2}$, and $q_{3}$ were specified directly by the user on the data line of the "POROUS METAL PLASTICITY" option. For typical metals the ranges of the parameters reported in the literature are $q_{1}=1.0-1.5, q_{2}=1.0$, and $q_{3}=1.0-2.25$ (HKS 2001). The original Gurson model is recovered when $q_{1}=q_{2}=q_{3}=1.0$. Because the original material was assumed to be undamaged, the initial void volume fraction was assigned to zero.

The damage evolves via the growth of existing voids and the nucleation of new voids. The porous metal plasticity model in ABAQUS computed the void growth automatically. The void nucleation was assumed to be strain-controlled (see Section 3.5). As described by Saje et al. (1982), the plastic strain controlled nucleation was described by the volume fraction $f_{\mathrm{n}}=0.04$ of void nucleating particles, the mean strain for nucleation $\varepsilon_{\mathrm{n}}=0.3$, and the standard deviation $s_{\mathrm{n}}=0.1$ in Eq. (8).

\subsection{Results}

\subsubsection{Elastic constants}

The degraded elastic constants due to damage evolution could be calculated by the following two steps. First, we obtained the damage values against the true axial strains from the finite element results. Thereafter, Eq. (3) was adopted to calculate the degraded elastic constants. Once the residual bulk modulus and shear modulus were determined, Young's modulus $E$ could be calculated by the relation:

$$
E=\frac{9 K G}{3 K+G} \text {. }
$$

The solid line in Fig. 9 represents the normalized residual Young's moduli calculated by the aforementioned method as a function of the true axial strain.

Both numerical and experimental data show that Young's moduli decrease with the increase in the applied axial strain and stiffness reduction begins early in the deformation process. As depicted in Fig. 9, the agreement of the calculated values with the experimental results is quite good (the maximum difference is about $1.3 \%$ ). It is shown that the finite element model can represent the actual material behavior with confident precision. Both the calculated and experimental values at the axial strain $\varepsilon=0.43$ show about a $10 \%$ reduction in Young's modulus. The finite element results indicate that further loading will lower the stiffness continuously.

Meanwhile, the damage value of each damaged tensile specimen could be computed by coupling the measured Young's modulus with Eqs. (3) and (11). The damage values obtained from the measured Young's moduli are shown in Fig. 11. By coupling Fig. 9 with Fig. 11, we can deduce that the characteristic of the damage evolution process dominates the decreasing feature of residual stiffness.

\subsubsection{Ultrasonic velocities}

After the residual elastic constants were determined by the finite element simulation, we utilized Eq. (9) to calculate the L-wave velocity of the strain-damaged materials. Fig. 10 shows the L-wave velocities as a function of the true axial strain. The solid line represents the predicted values of L-wave velocities by finite element simulation, and the square symbols are the experimental values. The simulated and measured 


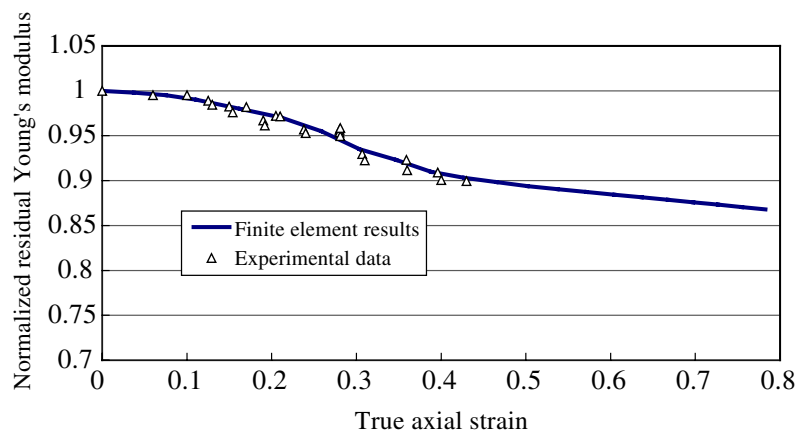

Fig. 9. Comparison of experimental and simulated stiffness vs. true strain curves.

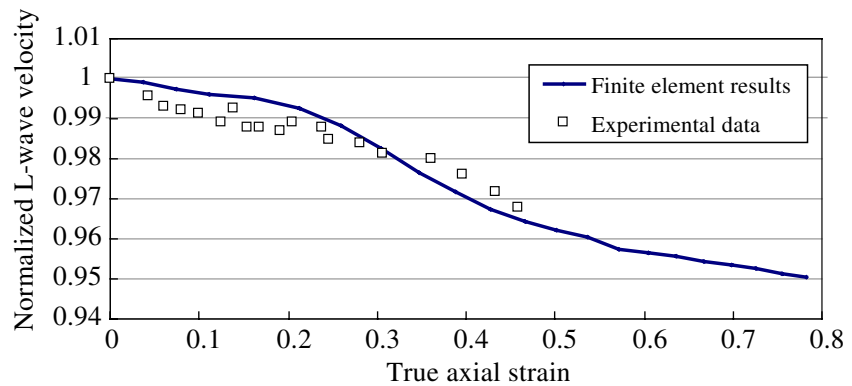

Fig. 10. Comparison of experimental and simulated velocities of L-waves vs. true strain curves.

velocities of the original specimen are 5723.7 and $5724 \mathrm{~m} / \mathrm{s}$, respectively. Both predicted and experimental results show that L-wave velocities decrease with the axial strain. Although the variation in the ultrasonic velocity is not as significant as that in Young's modulus, the decreasing trend is quite obvious. Both the calculated and experimental values at axial strain $\varepsilon=0.46$ show about a $3.5 \%$ reduction in L-wave velocity.

As depicted in Fig. 10, the experimental results deviate from the computed ones slightly. This may be caused by the preparation of the ultrasonic samples, which were made by cutting from the tensile specimens and surface polishing (see Section 5.3). The extra machining induced a slight change of the damage state, which could not be predicted by the finite element model. The effect of the extra machining is less significant in larger deformation.

\subsubsection{Comparison}

The damage values of the loaded specimen can be obtained from the finite element simulation, the measurement of residual Young's moduli, and the measurement of the reduced L-wave velocities. The damage evolutional process is described in Fig. 11. The results of the finite element simulation agree with those obtained from both the tensile tests and the ultrasonic tests. From the finite element results shown in Fig. 11, the damage increases rapidly in the early stage of the deformation; however, the increase in the damage becomes slower as the axial strain exceeds about 0.45. By coupling Fig. 11 with Figs. 9 and 10, it can be concluded that the increasing feature of the damage variable affects directly the evolving trends of the elastic constants and the L-wave velocities.

From the above analysis, we prove that the proposed elastoplastic damage model can precisely represent the processes of the deformation and damage evolution of metals, and predict the reductions in the elastic 


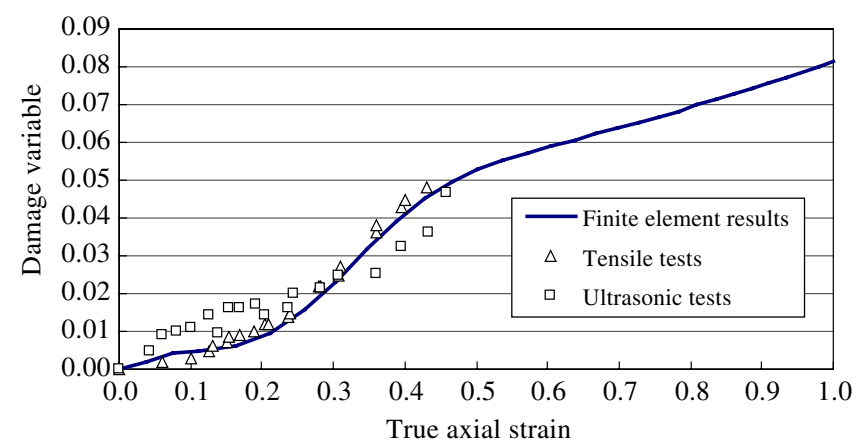

Fig. 11. Damage variables obtained from simulation, tensile tests, and ultrasonic tests.

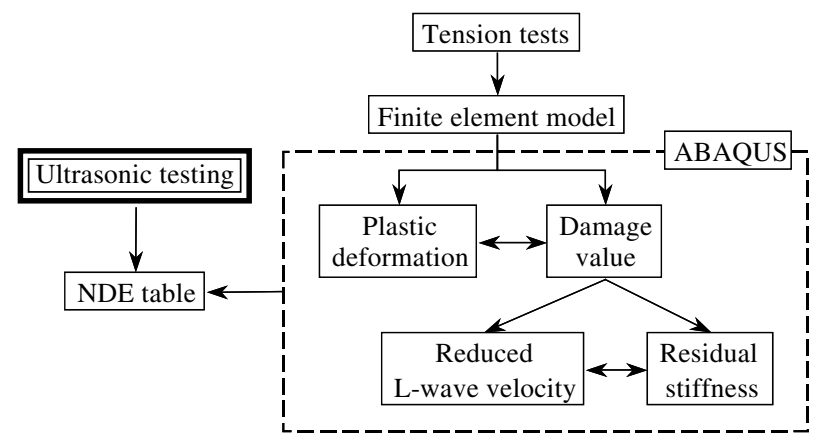

Fig. 12. Schematic diagram of the nondestructive damage evaluation procedure.

constants and the L-wave velocities as a function of the damage variable. Therefore, ultrasonic pulse-echo method is suitable for the nondestructive evaluation of metal damage.

\subsection{Discussion}

The above procedure illustrates a novel nondestructive method for quantitative evaluation of metal damage. As shown in Fig. 12, for any newly received material, one needs to begin with tension tests to acquire the mechanical properties for use in the finite element model. If the mechanical properties and the flow stress-strain data are readily available, this step can be skipped. Based on the finite element results, we can create a table of the correlations between the damage variable, plastic deformation, residual stiffness, and the NDT parameter (defined as the reduction rate of L-wave velocity, $\Delta v / v_{0}$ ). With this table or chart, the proposed approach can be adopted to other applications, be it structural or during a metal forming process. By just employing the ultrasonic pulse-echo method to estimate the L-wave velocity of the damaged material and consulting the aforementioned damage table, we can obtain the current damage value, from which other residual mechanical properties can be ascertained.

\section{Conclusions}

By correlating the ultrasonic pulse-echo method to the proposed elastoplastic damage model, we have successfully established a novel nondestructive damage assessment technique. From the results shown above, we can draw the following conclusions: 
(1) We propose a new concept of micromechanics based damage model. Only one scalar damage variable is needed, and the mechanical behavior of damaged materials is estimated with the help of Mori-Tanaka method.

(2) By adopting the finite element method with the proposed damage model, processes of deformation and damage evolution are precisely predicted, from which the reductions in elastic constants and L-wave velocities can be predicted.

(3) Since the correlations between the damage value, plastic strain, residual mechanical properties and NDT parameter can be established by the finite element model, we just employ the ultrasonic pulseecho method to estimate the L-wave velocity of the damaged material and can obtain the current damage state by means of the previously developed data bank.

(4) The proposed nondestructive damage evaluation technique can be extended to a scanning configuration to map out local damage in the metal forming process.

\section{Acknowledgement}

This research has been supported by National Science Council of Taiwan under grant NEC 91-2212-E002-039.

\section{References}

Augereau, F., Roque, V., Robert, L., Despaux, G., 1999. Non-destructive testing by acoustic signature of damage level in 304L steel samples submitted to rolling, tensile test and thermal annealing treatments. Materials Science and Engineering A 266, $285-294$.

Birks Jr., A.S., Green, R.E., 1991. Ultrasonic Testing. American Society for Nondestructive Testing, Columbus.

Brown, L.M., Embury, J.D., 1973. The initiation and growth of voids at second phase particles. In: Proceedings of 3rd International Conference on Strength of Metals and Alloys, London, pp. 164-169.

Chaboche, J.L., 1979. Le concept de contrainte effective appliqué à I'elasticité et à la viscoplasticité en présence d'un endommagement anisotrope. In: Comportement Mécanique des Solides Anisotropes. EUROMECH Colloque. Martinus Nijhoff 115, 737-760.

Chu, C.C., Needleman, A., 1980. Void nucleation effects in biaxially stretched sheets. Journal of Engineering Materials and Technology 102, 249-256.

Gruber, J.J., Smith, J.M., Brockelman, R.H., 1988. Ultrasonic velocity $C$-scans for ceramic and composite material characterization. Materials Evaluation 46 (1), 90-96.

Gurson, A.L., 1977. Continuum theory of ductile rupture by void nucleation and growth: Part I-yield criteria and flow rules for porous ductile media. Journal of Engineering Materials and Technology, Transactions of the ASME 99 (1), 2-15.

Hibbitt, Karlsson and Sorenson, Inc., 2001. ABAQUS/Standard User's Manual, Version 6.2.

Hult, J., 1974. Creep in continua and structures. In: Topics in Applied Continuum Mechanics. Springer, New York, p. 137.

Jaunzemis, W., 1967. Continuum Mechanics. The Macmillan Co., New York.

Jeong, H., Hsu, D.K., 1996. Quantitative estimation of material properties of porous ceramics by means of composite micromechanics and ultrasonic velocity. NDT\&E International 29 (2), 95-101.

Kachanov, L.M., 1958. On creep rupture time. Otdelenie Tecknicheskikh Nauk, Izvestiya Akademii Nauk SSSR 8, $26-31$.

Krajcinovic, D., Lemaitre, J., 1987. Continuum Damage Mechanics Theory and Applications. Springer-Verlag, Wien.

Leckie, F.A., Hayhurst, D., 1974. Creep rupture of structures. Proceedings of the Royal Society London A 340, $323-347$.

Lemaitre, J., 1971. Evaluation of dissipation and damage in metals submitted dynamic loading. In: Proceedings of ICM1, Kyoto.

Lemaitre, J., 1985. A continuous damage mechanics model for ductile fracture. Journal of Engineering Materials and Technology 107, $83-89$.

Lemaitre, J., 1992. A Course on Damage Mechanics. Springer-Verlag, Berlin.

Lemaitre, J., Chaboche, J.L., 1990. Mechanics of Solid Materials. Cambridge University Press, London.

Malvern, L.E., 1969. Introduction to the Mechanics of a Continuous Medium. Prentice-Hall, Englewood Cliffs.

Maugin, G.A., 1992. The Thermomechanics of Plasticity and Fracture. Cambridge University Press, Cambridge.

Mori, T., Tanaka, K., 1973. Average stress in matrix and average energy of materials with misfitting inclusion. Acta Metallurgica 21, $571-574$. 
Nagarajan, A., 1971. Ultrasonic study of elasticity-porosity relationship in polycrystalline alumina. Journal of Applied Physics 42 (10), 3693-3696.

Panakkal, J.P., Ghosh, J.K., Roy, P.R., 1984. Use of ultrasonic velocity for measurement of density of sintered fuel pellets. Journal of Physics D. Applied Physics 17, 1791-1795.

Rabotnov, Y.N., 1968. Creep rupture. In: Proceedings of the XII International Congress on Applied Mechanics, pp. $342-349$.

Saje, M., Pan, J., Needleman, A., 1982. Void nucleation effects on shear localization in porous plastic solids. International Journal of Fracture 19 (3), 163-182.

Truesdell, C., Noll, W., 1965. The nonlinear field theories of mechanics. In: Flugge, S. (Ed.), Encyclopedia of Physics III/3. SpringerVerlag, Berlin.

Tvergaard, V., 1981. Influence of voids on shear band instabilities under plane strain conditions. International Journal of Fracture 17 (4), 389-407.

Tvergaard, V., Needleman, A., 1984. Analysis of the cup-cone fracture in a round tensile bar. Acta Metallurgica 32 (1), $157-169$.

Jung-Ho Cheng: Past experience: M.S. and Ph.D. of Mechanical Engineering, The University of Michigan, Ann Arbor, Michigan, USA (1980-1984); Research Engineer of Cooperate Research and Development Center, General Electric Company, Schenectady, New York, USA (1985-1990); Associate Professor of Mechanical Engineering, National Taiwan University, Taipei, Taiwan (1990-1996). Research interests: Composite Materials, Damage Mechanics, Biomechanics, Wind Energy, Solar Vehicle, Fuel Cell, Hydrogen-Energy Society, Finite Element Methods, Metal Forming, Tire Mechanics. 